\title{
CLASSICAL LIMIT OF ENTANGLED CORRELATIONS
}

\author{
K. WóDKIEWICZ* \\ Institute of Theoretical Physics, Warsaw. University, Hoża 69, 00-681 Warszawa, Poland
}

\begin{abstract}
Generalizations of Bell's inequalities for Einstein Podolsky and Rosen entangled correlations for arbitrary spin $s$ are discussed. Violations of local realism and the classical limit of quantum nonlocality for large $s$ are discussed. The role of the polarization and of the spin alignment in the violation of the Bell inequalities are investigated. A Bayes analysis of entangled correlations is performed using the nonlocal quantum distribution. The local but nonpositive Einstein Podolsky and Rosen quantum distribution is investigated in the limit when $s \rightarrow \infty$. The classical limit of the quantum Malus law for arbitrary spin $s$ is formulated in terms of the spin path integrals.
\end{abstract}

PᄉCS numbers: 42.50.Wm, 03.65.Bz

\section{Bell's inequalities for arbitrary spin $s$}

The concept of local realism (LR) is based on the fundamental assumption that physical systems can be described by local objective properties that are independent from observation. LR versus the quantum description has been described best in the framework of spin- $\frac{1}{2}$ Einstein Podolsky and Rosen (EPR) correlations [1]. The generalization of the EPR entangled correlations for arbitrary spin $s$ involves the measurements of the spin correlation $E(a ; b)$, and the spin transmission probability $p(a ; b)$. In quantum mechanics the spin correlation is

$$
E(a ; b)=\operatorname{Tr}\{\widehat{\rho} \widehat{s}(a) \otimes \widehat{s}(b)\}
$$

and the spin transmission probability is defined by the following formula:

$$
p(a ; b)=\operatorname{Tr}\{\hat{\rho} \widehat{P}(a) \otimes \widehat{P}(b)\} .
$$

In these expressions $\widehat{s}(a)(\widehat{s}(b))$ and $\widehat{P}(a)(\widehat{P}(b))$ are the spin and the projection operators of the particle $a(b)$ along the polarization direction $a(b)$ and $\hat{\rho}$ is the density operator that describes the entangled state of the two particles with arbitrary spin.

*Also associated with the Center for Advanced Studies and Department of Physics and Astronomy, University of New Mexico, Albuquerque, NM 87131, USA. 
Following the basic ideas of a theory based on LR the spin components measured by linear polarizers are objective realities described by deterministic functions $s\left(a, \lambda_{a}\right)$ and $s\left(b, \lambda_{b}\right)$, with hidden variables $\lambda_{a}$ and $\lambda_{b}$. These hidden parameters are randomly distributed with a positive and normalized distribution function

$$
\int \mathrm{d} \lambda_{a} \int \mathrm{d} \lambda_{b} P\left(\lambda_{a} ; \lambda_{b}\right)=1 \text { and } P\left(\lambda_{a} ; \lambda_{b}\right) \geq 0,
$$

which is local, i.e., is independent on the polarization directions $a$ and $b$. In the framework of such a theory the transmission of an arbitrary spin $s$ through a linear polarizer $a$ is described by an objective reality represented by the transmission function $t\left(a, \lambda_{a}\right)$.

In a theory based on local hidden variables (LHV), the spin variables and the transmission functions are local realities that depend on hidden or unknown parameters which are averaged out during a detection process. Such theories lead to the spin correlation and to the spin transmission in the form of the following statistical averages of local realities:

$$
E(\boldsymbol{a} ; \boldsymbol{b})=\int \mathrm{d} \lambda_{a} \int \mathrm{d} \lambda_{b} P\left(\lambda_{a} ; \lambda_{b}\right) s\left(a, \lambda_{a}\right) s\left(b, \lambda_{b}\right)
$$

and

$$
p(\boldsymbol{a} ; \boldsymbol{b})=\int \mathrm{d} \lambda_{a} \int \mathrm{d} \lambda_{b} P\left(\lambda_{a} ; \lambda_{b}\right) t\left(a, \lambda_{a}\right) \iota\left(b, \lambda_{b}\right) .
$$

If we assume that the objective realities $s\left(a, \lambda_{a}\right)$ and $s\left(b, \lambda_{b}\right)$ of the spin can take up only values from $-s$ to $s$, we obtain that the spin correlations (3a), evaluated for four different polarization axes, are restricted by the following spin Bell's inequality (SBI) valid for arbitrary spin $s$ [2]:

$$
\left|E(\boldsymbol{a} ; \boldsymbol{b})+E\left(\boldsymbol{a}^{\prime} ; \boldsymbol{b}\right)+E\left(\boldsymbol{a}^{\prime} ; \boldsymbol{b}^{\prime}\right)-E\left(\boldsymbol{a} ; \boldsymbol{b}^{\prime}\right)\right| \leq 2 s^{2} .
$$

If the transmission functions through the linear polarizers $a$ and $b$ are: $0 \leq t\left(a, \lambda_{a}\right) \leq 1$ and $0 \leq t\left(b, \lambda_{b}\right) \leq 1$, the transmission probabilities (1b) evaluated for four different polarization axes are restricted by the following transmission Bell's inequality (TBI) [3]:

$$
-1 \leq p(\boldsymbol{a} ; \boldsymbol{b})+p\left(\boldsymbol{a}^{\prime} ; \boldsymbol{b}\right)+p\left(\boldsymbol{a}^{\prime} ; \boldsymbol{b}^{\prime}\right)-p\left(\boldsymbol{a} ; \boldsymbol{b}^{\prime}\right)-p(\boldsymbol{a})-p(\boldsymbol{b}) \leq 0 .
$$

In this expression $p(a)$ and $p(b)$ are the marginals of the joint probability $p(a ; b)$. In analogy to quantum mechanics for particles with $s=1 / 2$, the objective transmission function and the objective spin reality are related by the equation

$$
t\left(a, \lambda_{a}\right)=1 / 2+s\left(a, \lambda_{a}\right)
$$

and in this case SBI and TBI are equivalent.

In a model LHV theory one can assume for example that the LR for the spin is $s\left(a, \lambda_{a}\right)=\frac{1}{2} \cos \alpha\left(a, \lambda_{a}\right)$, where $\alpha\left(a, \lambda_{a}\right)$ is the relative angle between $a$ and the objective spin orientation characterized by the hidden parameter $\lambda_{a}$. According to the relation (6a), the LR transmission function for the spin- $\frac{1}{2}$ particles is in this case

$$
t\left(a, \lambda_{a}\right)=\left(\cos \frac{\alpha}{2}\right)^{2} .
$$


This expression has the form of the well-known classical Malus law that predicts an attenuation of the light intensity through a linear polarizer.

For $s \geq 1 \mathrm{SBI}$ and TBI are not equivalent. The nonequivalence of these two Bell's type inequalities for $s \geq 1$ can be illustrated using the following argument. Consider the direct product of two irreducible representations of the rotation group. For the spin group we obtain in this case the following Clebsch-Gordan series: $D^{s} \otimes D^{s}=D^{0} \oplus D^{1} \oplus D^{2} \oplus \ldots \oplus D^{2 s}$. For spin- $\frac{1}{2}$ systems the only components in this series are the scalar and the vector representations. For this particular value of the spin we have the very simple relation between the spin and the projection operators: $\widehat{P}(a)=1 / 2+\widehat{s}(a)$. This relation involves the scalar and the vector components only. This relation is used in the expression (6a) in the LIIV theory for the objective realities of the spin and the transmission function. For arbitrary spin $s$ the relation between the spin and the projection operators is [4]

$$
\widehat{P}=\frac{1}{2 s+1} \sum_{s=0}^{2 s} \sum_{m=-s}^{s} q_{m}^{s} \widehat{Q}_{m}^{s}
$$

where $\widehat{Q}_{m}^{s}$ are irreducible tensors of rank $(s, m)$. The vector component of the projection operator is called the spin polarization term, while all higher tensors are called spin alignment. Only for spin- $\frac{1}{2}$ the vector component uniquely determines the projector operator, as no higher tensors appear in this case. Because of this, the transmission correlations (1b) and (3b) differ from the spin correlations (1a) and (3a) by the terms involving for $s \geq 1$ tensor correlations of the two spins. Due to these tensor correlations we have the following relation between TBI and SBI:

\section{$T B I \supset S B I$ for $s \geq 1$.}

This symbolic relation expresses the fact that for higher spins SBI reflects only the vector character of the spin correlations, while TBI contains all the higher tensor correlations of the spin alignment.

\section{Violation of Bell inequalities}

The EPR entangled state of the iwo particles each with spin $s$ is given by the density operator $\hat{\rho}=|\psi\rangle\langle\psi|$, where $|\psi\rangle$ is the singlet state of the two correlated spins. Using the explicit expression for the Clebsch-Gordan coefficient and the spin eigenstates $\left|m_{a} ; m_{b}\right\rangle$ we obtain

$$
|\psi\rangle=\sum_{m=-s}^{s} \frac{(-1)^{s+m}}{\sqrt{2 s+1}}|m ;-m\rangle .
$$

From these definitions we obtain that the reduced (marginal) density operators of the individual spins are

$$
\hat{\rho}_{a}=\operatorname{Tr}_{b} \hat{\rho}=\frac{1}{2 s+1} \widehat{I}_{a} \quad \text { and } \quad \hat{\rho}_{b}=\operatorname{Tr}_{a} \hat{\rho}=\frac{1}{2 s+1} \widehat{I}_{b} .
$$

For the EPR state with arbitrary $s$ the quantum spin polarization correlation is [5]

$$
E(a ; b)=-\frac{s(s+1)}{3} \cos \alpha
$$


where $\alpha$ is the relative angle between the two directions $a$ and $b$. For the coplanar geometry of the polarizers $\left(a \cdot b=b \cdot a^{\prime}=a^{\prime} \cdot b^{\prime}\right)$ a maximum violation of the SBI given by (4) is expected for $\alpha=\pi / 4$. Introducing (9) into (4), we obtain that the Bell inequality is violated if $\sqrt{2} / 3 \leq s /(s+1)$. A violation is obtained only for $s=\frac{1}{2}$. For $s \geq 1$ the SBI is not violated at all [5].

It is clear that in order to test local realism for $s \geq 1$ we need to use the TBI with spin alignment. In order to do that we need to calculate correlations of the projection operators with polarization and alignment terms (for $s \geq 1$ ). A simple and a compact expression for the projection operator can be obtained if spin-coherent states (Bloch states) $|\Omega\rangle=|\theta, \phi\rangle$ are used. The spin coherent states can be scen as directions on a unit sphere (Bloch sphere) [6]. The solid angle $\Omega$ in this state describes an angular orientation of the spin-coherent state on such a Bloch sphere. In this coherent-state phase-space, the direction of the polarizer $a$ can be represented by the solid angle $\Omega_{a}=\left(\theta_{a}, \phi_{a}\right)$ and accordingly $\hat{p}(a)=\left|\Omega_{a}\right\rangle\left\langle\Omega_{a}\right|$. The spin-coherent states form an overcomplete set of statcs on the Bloch sphere and the projection operator of the polarizer is complete in the following sense:

$$
\frac{2 s+1}{4 \pi} \int \mathrm{d} \Omega_{a}\left|\Omega_{a}\right\rangle\left\langle\Omega_{a}\right|=\widehat{I},
$$

where the integration is over the solid angle on the Bloch sphere. Using this formula and the EPR spin-s wave function given by (8a) we obtain [7]

$$
p(a ; b)=\frac{1}{2 s+1}\left(\sin \frac{\alpha}{2}\right)^{4 s}
$$

and

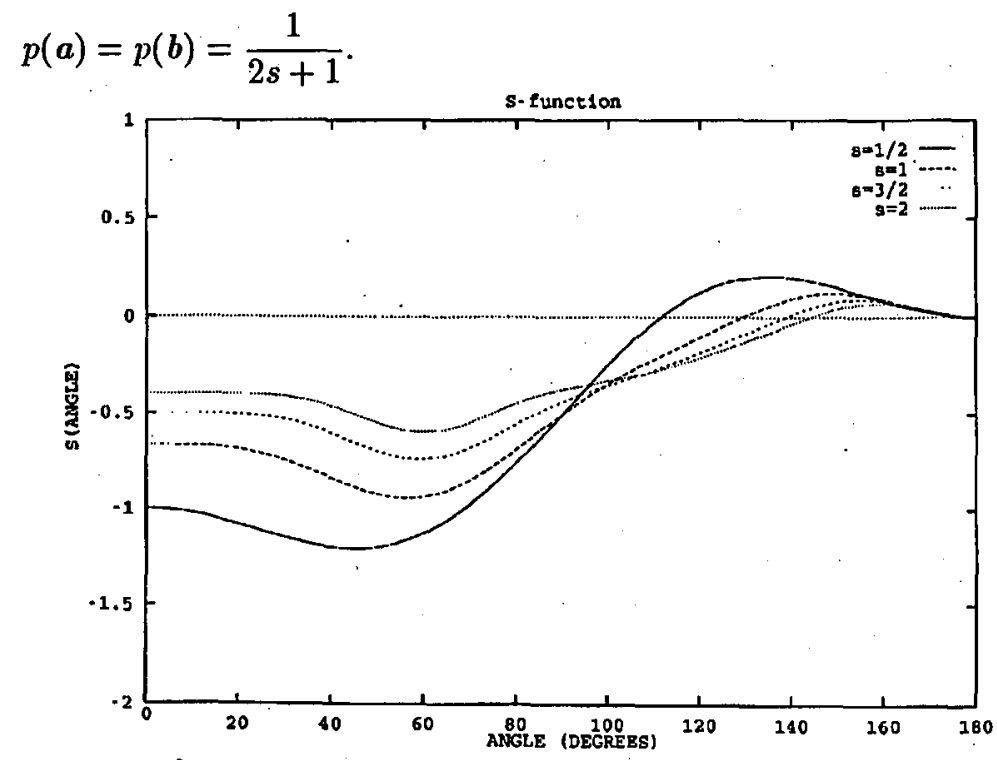

Fig. 1. Plots of $S$ as a function of the angle $\alpha$ for different values of $s$. 
This last relation expresses the fact that the EPR wave function if reduced to a single-spin subspace, becomes a completely mixed state. Using (11) (with a coplanar geometry for the polarizers) we obtain the TBI inequality (5) in the following form: $-1 \leq S(\alpha) \leq 0$, where the parameter $S$ is a function of the angle $\alpha$ only. In Fig. 1 there are plots of this function for different values of the spin $s$.

In contrast with SBI, TBI is violated for all values of $s$, although the violation decreases with increased values of $s$. This behavior is consistent with the expected classical behavior of the EPR correlations in the limit of $s \rightarrow \infty$.

\section{Classical limit of EPR correlations}

In the following we shall study the classical limit of the entanglement in the framework of quantum local and nonlocal correlations.

\subsection{Large spin limit of nonlocality}

We begin our discussion of the large spin limit of nonlocality using the two-point click counting distribution that has been introduced and applied recently to the discussion of quantum jumps in optical transitions [8]. Using the spectral decomposition of the spin projector operator

$$
\widehat{P}=\int \mathrm{d} \lambda \lambda \delta(\lambda-\widehat{P}),
$$

we can rewrite the quantum mechanical joint-probability (1b) in the following form:

$$
p(\boldsymbol{a} ; \boldsymbol{b})=\int \mathrm{d} \lambda_{a} \int \mathrm{d} \lambda_{b} \lambda_{a} \lambda_{b} P\left(a \lambda_{a} ; b \lambda_{b}\right),
$$

where the distribution function is given by the following quantum mechanical average:

$$
P\left(\boldsymbol{a} \lambda_{a} ; \boldsymbol{b} \lambda_{b}\right)=\operatorname{Tr}\left\{\hat{\rho} \delta\left(\lambda_{a}-\widehat{P}(\boldsymbol{a})\right) \otimes \delta\left(\lambda_{b}-\widehat{P}(\boldsymbol{b})\right)\right\} .
$$

With the help of this distribution function we have rewritten the quantum mechanical joint probability function (1b) in a form given by (13) which has remarkable similarities to the LHV correlation function given by Eq. (3b). Because the projection operators can have their eigenvalues equal to 1 or 0 , i.e., can represent only "yes" or "no" answers, the values $\lambda_{a}$ and $\lambda_{b}$ can take only values equal to 1 and 0 , i.e., corresponding to "clicks" at the detectors. The bivalued distribution given by Eq. (13) is positive everywhere, but depends on the polarization directions $a$ and $b$. The distribution function which depends on the orientation $a$ of the first analyzer and on the orientation $b$ of the second (possibly even remote) analyzer is nonlocal.

In the framework of EPR correlations it is customary to call an analyzer-dependent distribution function a nonlocal distribution function. The nonlocality of this distribution function makes the TBI inequality void, because in order to obtain this inequality the existence of a universal, local (polarization independent) distribution in the parameters $\lambda_{a}$ and $\lambda_{b}$ (hidden parameters in this case) is essential. 
Quantum mechanics tells us that if we insist on distribution of the form given by (13), we can do it but only under the condition that the statistical distribution of the parameters $\lambda_{a}$ and $\lambda_{b}$ is nonlocal.

In order to elucidate this point further, we shall perform a Bayes analysis of the two-point distribution function. The joint distribution can be written in the following form:

$$
P\left(\lambda_{a} ; \lambda_{b}\right)=P\left(\lambda_{a} \mid \lambda_{b}\right) P\left(\lambda_{b}\right) .
$$

The distribution $P\left(\lambda_{a} \mid \lambda_{b}\right)$ is the conditional of the event $\lambda_{a}$ ("yes" or "no") to occur under the condition that $\lambda_{b}$ ("yes" or "no") has occurred. From the definitions (13) and (8) we obtain that the single marginals of this joint distribution are

$$
P(0)=\frac{2 s}{2 s+1} \text { and } P(1)=\frac{1}{2 s+1} .
$$

The nonlocal conditional distribution is given by the following matrix:

$$
P\left(\lambda_{a} ; \lambda_{b}\right)=\left[\begin{array}{ll}
P(0 \mid 0) & P(0 \mid 1) \\
P(1 \mid 0) & P(1 \mid 1)
\end{array}\right]
$$

where

$$
P(0 \mid 0)=\frac{1}{2 s}\left[2 s-1+\left(\sin \frac{\alpha}{2}\right)^{4 s}\right] \text { and } P(0 \mid 1)=1-\left(\sin \frac{\alpha}{2}\right)^{4 s}
$$

with the sum rules fulfilled by the conditional probabilities

$$
P(0 \mid 0)+P(1 \mid 0)=1 \text { and } P(0 \mid 1)+P(1 \mid 1)=1 \text {. }
$$

In these formulas $\alpha$ is the relative angle between the two unit vectors $a$ and $b$. For notational convenience we have omitted the $\alpha$-argument in the distributions (16a). This result shows that one can regard the EPR correlations as just correlations of two sequences of random numbers represented by transmission functions $t\left(a, \lambda_{a}\right)=\lambda_{a}$ and $t\left(b, \lambda_{b}\right)=\lambda_{b}$ that are jumping between values 0 and 1 ("no" and "yes" answers) for polarization measurements performed with linear analyzers.

This positive and nonlocal distribution leads to a simple statistical interpretation of the spin transitions and of the violation of Bell's inequality in terms of random numbers 1 and 0 for the transmission functions. The quantum mechanical average in this case is represented by an ensemble average of two sequences of random numbers 1 and 0 . This random character of these variables can be applied in the description of the EPR correlations measured by two polarizers. To each polarizer there corresponds a sequence of random variables denoted by $t\left(a, \lambda_{a}\right)$ and $t\left(b, \lambda_{b}\right)$. These are the only possible outcome of the transmission experiment. On each single polarizer the outcomes are completely random and the "yes" and "no" answers occur with probabilities $P(0)$ for "no" and $P(1)$ for "yes" $(1 / 2$ in this case). The nonlocality of the EPR correlations shows up in the fact that these two perfectly random sequences (on the first and the second polarizers) are correlated and the correlations are given by Eq. (13a). These formulas predict that the EPR wave function can be understood as a nonlocal correlation between two random sequences $t\left(a, \lambda_{a}\right)=(1,0,0,1,1,0,0, \ldots)$ and $t\left(b, \lambda_{b}\right)=(0,0,1,1,0, \ldots)$. The nonlocality of these correlations follows from the fact that whenever $t\left(b, \lambda_{b}\right)=1$ on the polarizer $b$, we must have $t\left(a, \lambda_{a}\right)=1$ or 0 on the polarizer $a$ with the probabilities $P(1 \mid 1)$ and $P(0 \mid 1)$, i.e., the outcomes on $a$ (possibly even a remote 


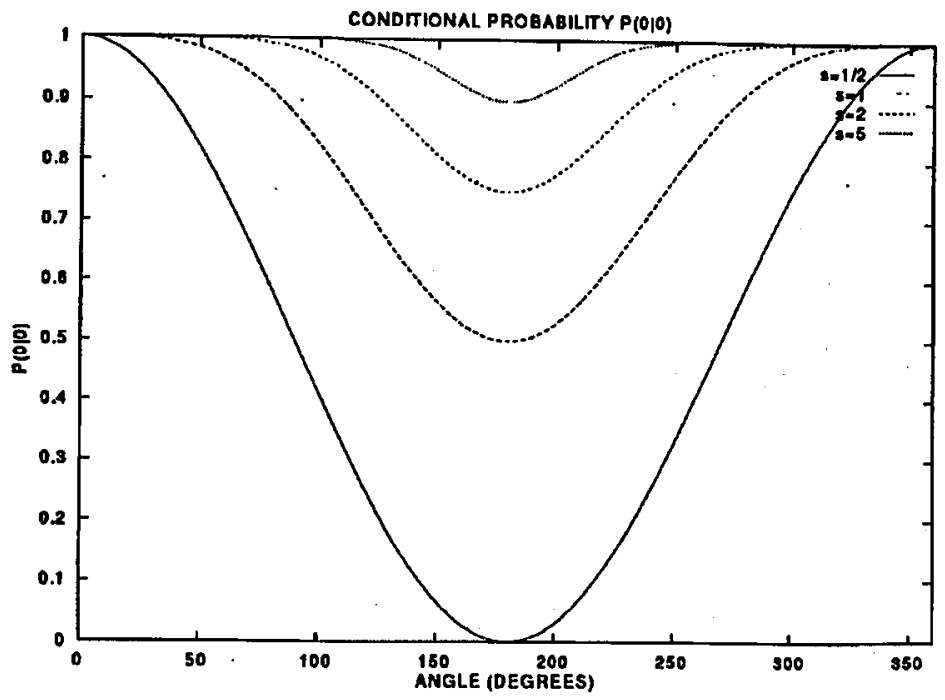

Fig. 2. Plots of the conditional probability $P(0 \mid 0)$ as a function of the angle $\alpha$ for different values of $s$.

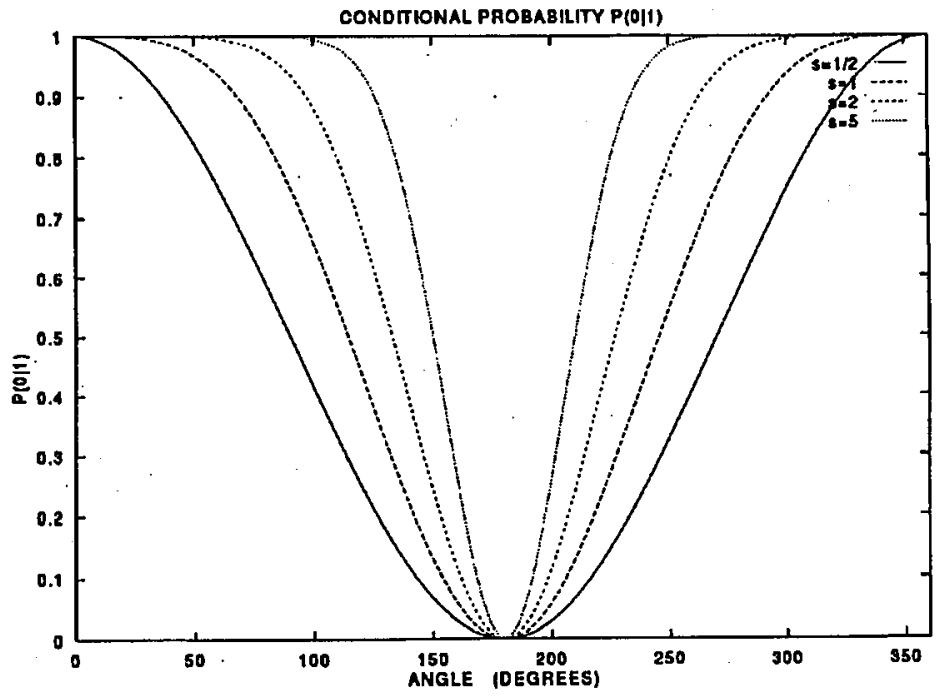

Fig. 3. Plots of the conditional probability $P(0 \mid 1)$ as a function of the angle $\alpha$ for different values of $s$.

analyzer) are determined by the outcomes on the analyzer $b$. This is how the EPR quantum sequences of random numbers violate local realism. In Fig. 2 and Fig. 3 there are plots of the conditional probabilities $P(0 \mid 0)$ and $P(0 \mid 1)$ as functions of the angle $\alpha$ for different values of the spin $s$. It is clear from this figures that with 
the increased value of the spin $s$ these distribution functions become sharper closer to only "yes" or "no" answers.

Let us investigate, using these random sequences, the large spin limit of nonlocality. In the limit of $s \rightarrow \infty$ we have

$$
\begin{aligned}
& P\left(\lambda_{a} \mid \lambda_{b}\right)=\left[\begin{array}{ll}
1 & 1 \\
0 & 0
\end{array}\right] \quad \text { for } \alpha \neq \pi, \\
& P\left(\lambda_{a} \mid \lambda_{b}\right)=\left[\begin{array}{ll}
1 & 0 \\
0 & 1
\end{array}\right] \text { for } \alpha=\pi .
\end{aligned}
$$

This means that for $\alpha=\pi$ we have a $100 \%$ confidence that the outcome on $a$ will be the same as the outcome on $b$. For $\alpha \neq \pi$ we now have $0 \%$ confidence that the outcomes on $a$ will be the same as the outcomes on $b$. This is precisely what we can expect from the entangled correlations in the classical limit corresponding to $s \rightarrow \infty$. In this limit the quantum nonlocal distribution becomes a local probability distribution corresponding to sharp "yes" and "no" outcomes of the measurement involving classical antiparallel angular momenta. From (11) we obtain that the conditional probability for spin transmission is

$$
p(a \mid b)=p(a ; b) / p(b)=\left(\sin \frac{\alpha}{2}\right)^{4 s}
$$

and

$$
\lim _{s \rightarrow \infty} p(a \mid b)=\left\{\begin{array}{lll}
\text { "always" } & \text { for } \alpha=\pi \\
\text { "never" } & \text { for } \quad \alpha \neq \pi .
\end{array}\right.
$$

\subsection{Spin quasi-distributions and the classical limit}

In full analogy to the coherent state diagonal $P$-representation for bosons, an arbitrary density matrix $\hat{\rho}$ of the spin system can be represented in the following diagonal form [9]:

$$
\hat{\rho}=\int \mathrm{d} \lambda_{a} \int \mathrm{d} \lambda_{b} P\left(\lambda_{a} ; \lambda_{b}\right)\left|\lambda_{a}, \lambda_{b}\right\rangle\left\langle\lambda_{a}, \lambda_{b}\right|,
$$

where the diagonal weight function $P\left(\lambda_{a}, \lambda_{b}\right)$ has the meaning of a quasi-probability distribution, and accordingly contains all the statistical information about the spin state. In this expression the hidden parameters correspond to the random orientations of the spin system in the phase-space generated by the spin coherent states, i.e., $\lambda_{a}=\Omega_{a}^{\prime}, \lambda_{b}=\Omega_{b}^{\prime}$.

From this relation, we obtain that the probability of transmission through a polarizer of an arbitrary spin state is a statistical average of the form (3b), and is given by the following formula:

$$
P(\boldsymbol{a} ; \boldsymbol{b})=\left.\int \mathrm{d} \lambda_{a} \int \mathrm{d} \lambda_{b} P\left(\lambda_{a} ; \lambda_{b}\right)\left|\left\langle\lambda_{a}\right| \Omega_{a}\right)\right|^{2}\left|\left\langle\lambda_{b} \mid \Omega_{b}\right\rangle\right|^{2} .
$$

The direction of the polarizer $a$ is described by a state with an orientation given by the solid angle $\Omega_{a}$ and accordingly the quantum probability of the spin transmissions through such polarizer is given by the following formula:

$$
t\left(a, \lambda_{a}\right)=\left|\left\langle\lambda_{a} \mid \Omega_{a}\right\rangle\right|^{2}=\left(\cos ^{2} \frac{\alpha_{a}}{2}\right)^{2 s},
$$


where $\cos \alpha_{a}=\cos \theta_{a}^{\prime} \cos \theta_{a}+\sin \theta_{a}^{\prime} \sin \theta_{a} \cos \left(\phi_{a}^{\prime}-\phi_{a}\right)$ gives the relative spherical angle between the hidden orientation $\lambda_{a}$ of the detected state and the direction $\Omega_{a}$ of the measuring polarizer. $A$ similar expression holds for the transmission function $t\left(b, \lambda_{b}\right)$. The quantum mechanical formula (20) has the form of a LHV theory with local realities given by $\cos \alpha_{a}$ and $\cos \alpha_{b}$ and that the "hidden-variable" directions $\Omega_{a}^{\prime}$ and $\Omega_{b}^{\prime}$ are integrated out. In this quantum mechanical expression the formula for the transmission function (21) provides a generalization of the Malus law (6b) to the case of an arbitrary spin $s$. In the following we shall, call these expressions spin Malus transmission functions.

From Eq. (19) we can derive that the EPR entangled state of two correlated spins is described by the distribution function which can be written in terms of the Legendre polynomials [10]

$$
P\left(\lambda_{a}, \lambda_{b}\right)=\sum_{l=0}^{2 s} c_{l} P_{l}(\cos (\alpha)) .
$$

In this formula $\cos \alpha$ describes the relative angle between the two hidden directions $\lambda_{a}$ and $\lambda_{b}$ on the unit Bloch sphere. The coefficients $c_{l}$ are calculated from the explicit form of the entangled wave function (8a). In Fig. 4 there are plots of this distribution function as functions of the angle $\alpha$ for different values of the spin $s$. From this form it follows that the EPR quasi-distribution function can be

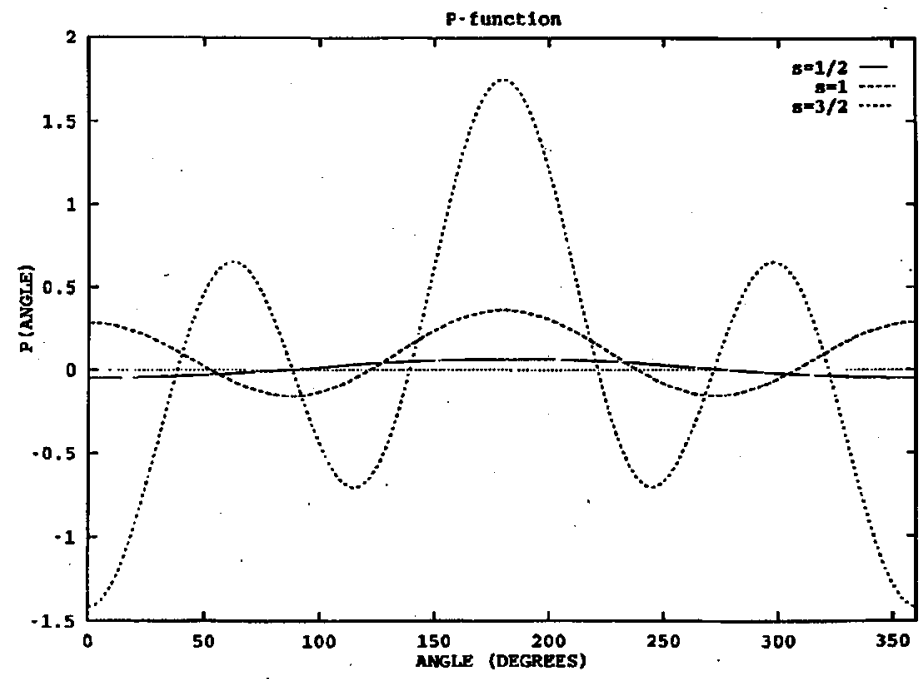

Fig. 4. Plots of the probability $P\left(\lambda_{a}, \dot{\lambda_{b}}\right)$ as a function of the angle $\alpha$ for different values of $s$.

negative and is independent from the directions of the polarizers used to detect the two spins. This means that this distribution function is local, in the sense used in the LIV theory. The only fundamental difference is that this distribution function can be negative making the Bell inequality inapplicable in this case. 
As the classical limit $s \rightarrow \infty$ is approached, the nonpositive local probability distribution (22) tends to a positive and local distribution of the following form:

$$
\lim _{s \rightarrow \infty} P\left(\lambda_{a}, \lambda_{b}\right)=\frac{1}{4 \pi} \delta^{(2)}\left(\lambda_{a}+\lambda_{b}\right)
$$

This expression corresponds to a sharp distribution of the classical antiparallel angular momenta.

\subsection{Classical limil of the quantum Malus law. The spin path integral}

Following the formula (21) we see that the quantum amplitude for the transmission of a spin state $\left|\Omega_{0}\right\rangle$ through a linear polarizer characterized by $\left|\Omega_{a}\right\rangle$ is: $\mathcal{A}=\left\langle\Omega_{a} \mid \Omega_{0}\right\rangle$ and that the probability is just the quantum Malus transmission function $t\left(a, \lambda_{a}\right)=\left|\left\langle\Omega_{0} \mid \Omega_{a}\right\rangle\right|^{2}$. Following the basic idea of path integration, we shall evaluate the Malus amplitude dividing the spin trajectory on the Bloch sphere into infinitesimal subintervals $\left|\Omega_{i}\right\rangle$, where $i=1, \ldots, N$ with $\Omega_{1}=\Omega_{0}$ and $\Omega_{N}=\Omega_{a}$. Using the decomposition of unity (10) for the spin coherent states for each subinterval and the infinitesimal form of the Malus amplitude $\left\langle\Omega_{i} \mid \Omega_{i-1}\right\rangle$ we obtain

$$
\begin{aligned}
\mathcal{A}= & \int \mathrm{d} \Omega_{1} \frac{2 s+1}{4 \pi} \int \mathrm{d} \Omega_{2} \frac{2 s+1}{4 \pi}, \\
& \ldots \exp \left(-\mathrm{i} s \sum_{i}\left(\phi_{i}-\phi_{i-1}\right) \cos \theta_{i-1}\right) .
\end{aligned}
$$

In the limit of $N \rightarrow \infty$ this expression can be written in the form of the spin Feynman path integral

$$
\mathcal{A}=\int \mathcal{D} \Omega \frac{2 s+1}{4 \pi} \exp \left(-\mathrm{i} s \int \mathrm{d} \phi \cos \theta\right),
$$

where $\mathcal{D} \Omega$ is the functional path integration measure over all trajectories connecting $\left|\Omega_{0}\right\rangle$ with $\left|\Omega_{a}\right\rangle$ on the Bloch sphere. Upon the following identification:

$$
\phi \Leftrightarrow q, \quad \cos \theta \Leftrightarrow p, \quad \mathrm{~d} \phi \mathrm{d} \cos \theta \Leftrightarrow \mathrm{d} q \mathrm{~d} p,
$$

we can rewrite the path integral (24) in the form

$$
\mathcal{A}=\int \mathcal{D} q \int \mathcal{D} p \frac{2 s+1}{4 \pi} \exp \left(-\mathrm{i} s \int p \mathrm{~d} q\right)
$$

which is the spin analog of the phase-space path integral for the following quantum mechanical amplitude in the configuration space:

$$
\left\langle q_{a} \mid q_{0}\right\rangle=\int \mathcal{D} q \int \mathcal{D} p \frac{1}{2 \pi \hbar} \exp \left(-\frac{\mathrm{i}}{\hbar} \int \mathrm{d} q p\right) .
$$

The Malus probability for a spin transition of the state $\left|\Omega_{0}\right\rangle$ through a linear polarizer can be expressed as a product of four path integrals

$$
\begin{aligned}
|\mathcal{A}|^{2} & =\int \mathcal{D} q_{1} \int \mathcal{D} p_{1} \frac{2 s+1}{4 \pi} \int \mathcal{D} q_{2} \\
& \times \int \mathcal{D} p_{2} \frac{2 s+1}{4 \pi} \exp \left(\mathrm{i} \mathcal{S}\left(q_{1}, p_{1}\right)-\mathrm{i} S\left(q_{2}, p_{2}\right)\right)
\end{aligned}
$$


where the classical action is

$$
\mathcal{S}(q, p)=s \int p \mathrm{~d} q+\frac{1}{\hbar} \int \mathcal{H} \mathrm{d} t
$$

In this expression a classical IIamiltonian $\mathcal{H}$ has been added in order to describe a transition through the linear polarizer with a dynamical time evolution. In the limit of $s \rightarrow \infty$, with the following change of coordinates in the path integrals: $q_{1,2}=q \pm(1 / 2 s) \tilde{q}, \quad p_{1,2}=p \pm(1 / 2 s) \tilde{p}$, the Malüs transmission function reduces to $[10]$

$$
\lim _{s \rightarrow \infty}|\mathcal{A}|^{2}=\int \mathcal{D} q \int \mathcal{D} p \delta\left(\dot{q}-\frac{1}{s \hbar} \frac{\partial \mathcal{H}}{\partial p}\right) \delta\left(\dot{p}+\frac{1}{s \hbar} \frac{\partial \mathcal{H}}{\partial q}\right)
$$

This expression shows that in the classical limit the Malus transmission function reproduces the classical dynamics of an angular momentum with the following equations of motion:

$$
\dot{\phi}=\{\phi, \mathcal{H}\} \text { and } \dot{\theta}=\{\theta, \mathcal{H}\},
$$

where the Poisson bracket of the classical dynamics is given by the following formula:

$$
\{A, B\}=\frac{1}{s \hbar \sin \theta}\left(\frac{\partial A}{\partial \phi} \frac{\partial B}{\partial \theta}-\frac{\partial A}{\partial \theta} \frac{\partial B}{\partial \phi}\right) .
$$

\section{Conclusions}

In this paper generalizations of the Bell inequalities for EPR correlations for arbitrary spin $s$ liave been discussed. Violations of local realism and the classical limit of the EPR correlations for large $s$ has been investigated in the framework of local and nonlocal correlations. The relations (13) and (20) show that the violation of Bell's incquality can be due either to nonpositive and local or to positive and nonlocal distribution functions. The local and nonlocal probabilitics in FPR correlations are similar to the one encountered if one uses colserent states to study the harmonic oscillator problem. It is known that in this case the same results can be obtained with different quasi-distributions (the so-called $P$ - or $Q$-representations, for example). This shows that quantum mechanics is equivalent to a hidden-variable theory with nonpositive quasi-probabilities or to a lidden-variable theory with nonlocal distribution functions. Which view we adopt is quite irrelevant because the two pictures are equivalent and represent different aspects of the same quantum mechanical reality. In the classical limit the EPR entangled correlations lead to only "yes" or "no" outcomes involving classical antiparallel angular momenta. In this limit the local and the nonlocal quasi-distribution functions become positive and local statistical distributions.

\section{Acknowledgments}

This work was partially supported by the grants 2042699101 and 204279101 of the Committee for Scientific Research. 


\section{References}

[1] For review see M. Redhead, Incompleteness Nonlocality and Realism, Clarendon, Oxford 19.87.

[2] For $s=\frac{1}{2}$ see J. Bell, Physics 1, 195 (1964).

[3] J.F. Clauser, M.A. Horne, Phys. Reù. D 10, 526 (1974).

[1] See, for example, J.'Werle, Relativistic Theory of Reactions, Wiley, Now York 1966.

[5] N.D. Mermin, Phys. Rev. D 22, 356 (1980).

[6] See, for example, J.R. Klauder, B.S. Skagerstam, Colterent States, World Scientific, Singapore 1985.

[7] K. Wódkiewicz, in: Santa Fe Workshop of Foundations of Quanlum Mechanics, Eds. T.D. Black, M.M. Nieto, H.S. Pillof, M.O. Scully, R.M. Sinclair, World Scientific, Singapore 1992, p. 276.

[8] K. Wódkiewicz, J.H. Eberly, Ann. Phys. (NY) 216, 268 (1992).

[9] M.O. Scully, K. Wódkiewicz, in: Coherence and Quanium Optics VI, Eds. J.II. Eberly, L. Mandel, E. Wolf, Plenum Press, New York 1990, p. 1047.

[10] K. Wódkiewicz, to be published. 